\title{
Didactique des Sciences de la Vie et de la Terre en Formation Professionnelle des Enseignants et ses Impacts sur la Qualité de l'Enseignement au Maroc
}

\author{
Maouni Abdelfettah \\ Enseignant Chercheur, Agrégé en SVT, Coordonnateur d'une Filière \\ d'Education en SVT et Coordonnateur et Enseignant de Module de
} Didactique en SVT, Structure de Recherche « Biologie, Environnement et Développement Durable », Département des Sciences de la Matière et de la Vie, École Normale Supérieure de Tétouan, Université Abdelmalek Essaadi, Tétouan, Maroc

\section{Elhaboussi Mouad}

Professeur de l'Enseignement Secondaire Qualifiant en SVT, Doctorant, Structure de Recherche «Biologie, Environnement et Développement Durable », Département des Sciences de la Matière et de la Vie, École Normale Supérieure de Tétouan, Université Abdelmalek Essaadi, Tétouan, Maroc

\section{Saidi Rabah}

Enseignant chercheur, Agrégé en SVT, Enseignant de module de Didactique en SVT, Structure de Recherche «Biotechnologie Végétale », Département des Sciences de la Matière et de la Vie, École Normale Supérieure de Tétouan, Université Abdelmalek Essaadi, Tétouan, Maroc

\section{Résumé}

La didactique appliquée est la base de la formation dans le métier d'enseignement d'une discipline. En Sciences de la Vie et de la Terre (SVT), les leçons ou leçons TP encadrées selon la nouvelle vision de la didactique représentent l'avantage de bien préparer les élèves professeurs aux métiers d'enseignement et par conséquent d'améliorer la qualité de notre enseignement. La présente étude a été établie dans le but de valoriser la didactique appliquée en formation initiale et continue. Les résultats de recherche d'évaluation de la didactique appliquée au niveau des centres de formation nous ont permis de retenir que l'horaire consacré au module de la didactique appliquée est généralement insuffisant et que le contenu n'est pas totalement conforme à la nouvelle vision, dans la majorité des descriptifs universitaires d'Éducation. L'évaluation des élèves professeurs en séances de didactique appliquée en SVT montre que la plupart des étudiants ignorent 
totalement la préparation des fiches scientifiques et pédagogiques et rencontrent des problèmes énormes dans la conception des fiches et la présentation orale ou écrite d'une leçon; une telle situation exige des formateurs multidisciplinaires et qualifiés. L'évaluation en situation réelle de classe de secondaire, nous a permis de dégager un ensemble d'anomalies dans le métier d'enseignement qui demande une formation continue plus consistante et mieux réfléchie en Didactique Appliquée. Enfin, il convient de signaler que la pratique de la didactique selon les points soulevés dans cette étude, dans les centres de formation des enseignants, pourrait avoir un impact positif sur l'amélioration de la qualité de notre enseignement secondaire.

Mots-clés : Didactique appliquée, SVT, Fiche scientifique, Fiche pédagogique, TIC

\title{
Didactics of the Sciences of Life and Earth in Professional Training of Teachers and its Impacts on the Quality of Education in Morocco
}

\begin{abstract}
Maouni Abdelfettah
Enseignant Chercheur, Agrégé en SVT, Coordonnateur d'une Filière d'Education en SVT et Coordonnateur et Enseignant de Module de Didactique en SVT, Structure de Recherche « Biologie, Environnement et Développement Durable », Département des Sciences de la Matière et de la Vie, École Normale Supérieure de Tétouan, Université Abdelmalek Essaadi, Tétouan, Maroc
\end{abstract}

\section{Elhaboussi Mouad}

Professeur de l'Enseignement Secondaire Qualifiant en SVT, Doctorant, Structure de Recherche « Biologie, Environnement et Développement Durable », Département des Sciences de la Matière et de la Vie, École Normale Supérieure de Tétouan, Université Abdelmalek Essaadi, Tétouan, Maroc

\section{Saidi Rabah}

Enseignant chercheur, Agrégé en SVT, Enseignant de module de Didactique en SVT, Structure de Recherche « Biotechnologie Végétale », Département des Sciences de la Matière et de la Vie, École Normale Supérieure de Tétouan, Université Abdelmalek Essaadi, Tétouan, Maroc 


\section{Abstract}

Applied didactic is the basic training in the teaching profession of any discipline. Lessons or practical works, according to the new vision of teaching, seeks to better prepare students teachers. This paper focuses on highlighting applied didactic in both initial and continuous training. Research evaluation results of applied didactics in centre's training has shown that the hourly volume of applied didactic is generally insufficient. It also shows that the content is not very compliant with the new vision for the majority of curriculum. Evaluation of student teachers according to the new vision of applied didactic in Life and Earth Sciences (LES) has shown that most students have completely ignored the preparation of the scientific and educational sheets. In addition, they have encountered enormous problems in the construction and presentation of their lessons. This situation requires multi-disciplinary and qualified trainers. Real classroom evaluation has enabled us to identify a set of anomalies in the teaching profession, which requires more continuous training in applied didactics. Majority of teachers do not master Information and Communication Technology (ICT), and they considered the integration of technology in their lessons anti-pedagogical. In recent years, the LES lessons taken by students has become a set of data and results experiments, helping students to differentiate essential from nonessential knowledge. In the absence of adequate didactic orientation, classical teaching methods and even erroneous educational and didactic representations will continue to circulate in our educational system. The gravity of all this is the indirect transfer of this classical way of teaching from practicing teachers to student teachers during internships. Lastly, it should be pointed out that the practice of didactics, according to the points raised in this study in teacher training centers, could have a positive impact on improving the quality of our secondary education.

Keywords: Applied didactic, Life and Earth Sciences, Scientific Sheet, Educational Sheet, ICT

\section{Introduction}

La didactique des sciences est une discipline qui permet aux élèves professeurs d'acquérir un ensemble de compétences pédagogiques, scientifiques et technologiques liées principalement à l'acte et à l'art d'enseigner. Elle se différencie de la pédagogie par la nature des connaissances à enseigner et à faire évoluer. La même vision est perçue par d'autres auteurs (Ndong, 2011). La place et le rôle de la pratique enseignante sont essentiels et c'est plus à la recherche d'en tenir compte, que l'inverse (Martinand, 1994b). En effet, l'utilisation des résultats de la recherche en 
didactique dans l'enseignement passe nécessairement par les enseignants donc par leur formation (Demounem \& Astolfi, 1996)

Le savoir scientifique et technologique et le savoir-faire pédagogique, piliers de cette discipline l'ont rendue particulière et plus exigeante de la part des formateurs exerçants.

Avec le temps, l'enseignement de cette discipline dans les centres de formation des enseignants a connu une évolution d'une part vers une didactique générale ou théorique confiée principalement aux didacticiens spécialistes de cette discipline et d'autre part vers une Didactique Appliquée, appelée encore micro-enseignement, confiée principalement aux enseignants de la matière.

La didactique générale est devenue assez riche par son contenu basé essentiellement sur l'utilisation récurrente des résultats de recherche en didactique comme, par exemple, l'histoire des concepts scientifiques, les obstacles épistémologiques, les approches pédagogiques, les stratégies d'enseignement, la transposition didactique, le triangle didactique, les méthodes d'évaluation, la taxonomie de Bloom, etc.

Par ailleurs, la didactique appliquée à la discipline, terme non encore bien assimilé, ni par des didacticiens, ni par des enseignants chercheurs spécialistes de la matière, s'impose de plus en plus avec ses aspects pratiques et professionnels d'enseignement.

La complexité de la pratique enseignante, comme par exemple en SVT, réside donc d'une part, au niveau de la préparation par les élèves professeurs du contenu scientifique et sa présentation pédagogique et technologique sous forme d'une leçon ou leçon-TP et d'autre part, au niveau de l'évaluation et de l'orientation adéquate des actes d'enseigner par les formateurs. Il est efficient de rappeler que l'objectif principal de la Didactique Appliquée est l'acquisition d'un ensemble de compétences garantissant la réussite des élèves professeurs dans le métier d'enseignement.

\section{Constats préliminaires}

Il convient de signaler que le système de formation initiale ou continue des enseignants marocains n'a pas pu faire évoluer convenablement la didactique des sciences vers une réelle didactique appliquée ou pratique enseignante. En conséquence, la didactique appliquée n'est pas assez bien valorisée et n'est pas assez bien pratiquée dans toutes les disciplines y compris SVT. Ceci est corroboré par un ensemble de constats préliminaires que nous citons ci-dessous :

- Dans la plupart des cursus de Licence Professionnelle (LP), Filières Universitaires d'Education (FUE), l'horaire consacré à la Didactique Appliquée est insuffisant ; 
- Confusion entre Didactique Générale ou Sciences de l'Éducation et Didactique Appliquée ou leçons ;

- Dans la plupart des cas, l'encadrement des séances de Didactique Appliquée dans les centres de formation n'est pas assuré par des professeurs expérimentés maîtrisant le savoir scientifique, les concepts didactiques-pédagogiques et les Technologies de l'Information et de la Communication (TICE) ;

- Dans la plupart des cas, le contenu et les objectifs du module de Didactique Appliquée à la discipline ne sont pas adéquats ;

- Les enseignants chercheurs des centres de formation apprécient partiellement la Didactique Appliquée, sous-entendu microenseignement, et demeurent réticents aux appellations «leçons ou leçon /TP »;

- L'encadrement assuré par les inspecteurs pédagogiques au niveau des établissements reste insuffisant en absence des formations continues assidues ;

- Prolifération et évolution de représentations pédagogiques et didactiques erronées chez des enseignants exerçant en classe et n'ayant pas actualisé leur savoir et savoir-faire ;

- Transfert de méthodologies d'enseignement classiques au cours des stages des élèves professeurs.

\section{Problématique et objectifs visés}

Notre motivation pour le sujet de l'article est donc issue d'une part, des constats préliminaires cités ci-dessus et d'autre part, de notre conscience de l'importance de la Didactique Appliquée dans les pratiques d'enseignement. À ce niveau, nous reformulons la problématique relative au sujet comme suit :

- Comment peut-on valoriser la Didactique Appliquée dans les centres de formation?

- Quelle est notre nouvelle vision concernant la Didactique Appliquée ? Autrement dit, quelles sont les compétences visées en didactique appliquée ? Et comment amener les élèves professeurs ou enseignants exerçant dans les établissements à acquérir convenablement les nouvelles compétences?

- Comment amener les formateurs responsables de la Didactique Appliquée à adopter cette nouvelle vision ?

- Afin de résoudre cet ensemble de problèmes liés au sujet, nous avons visé les objectifs suivants :

- Évaluer et discuter le problème de la Didactique au niveau des centres de formation et au niveau des établissements scolaires ; 
- Instaurer les actes d'enseigner dans les pratiques d'enseignement en SVT :

- Mettre en lumière l'importance de la Didactique Appliquée dans les cursus de formation ;

- Établir le contenu des fiches pertinentes pour enseigner à bon escient en SVT : Fiches Scientifiques, Fiches Pédagogiques, fiches des activités d'apprentissage et de production de l'élève, fiches des évaluations ;

- Démontrer l'importance de l'intégration des TIC dans la transposition didactique des supports scientifiques (documents ou animations) et la présentation des actes d'apprentissage ;

- Inciter à l'utilisation pédagogique du tableau, du TBI et du vidéoprojecteur ;

- Former les élèves professeurs ou enseignants exerçants dans les établissements à acquérir correctement les nouvelles compétences dans la préparation et la présentation des leçons ;

- Persuader les formateurs responsables de la didactique appliquée en SVT et autres disciplines à adopter la nouvelle vision de la Didactique Appliquée ;

- Inciter les responsables de l'éducation et de la formation à augmenter les enveloppes horaires des formations continues en Didactique Appliquée.

\section{MÉTHODOLOGIE}

\section{Évaluation au niveau des centres de formation}

L'étude d'évaluation a été réalisée au niveau « LP », sur la base de vingtdeux filières «FUE » accréditées en différentes matières à l'École Normale Supérieure de Tétouan-Université Abdelmalek Essaadi-Tétouan-Maroc (seize «FUE » Métiers d'Enseignement accréditées après 2014 et six «FUE » accréditées avant 2014). Une autre filière LP - Métier d'enseignement en SVT a été évaluée en 2015 pour accréditation dans une faculté appartenant à une autre université marocaine. L'évaluation des descriptifs de différentes filières porte essentiellement sur le module de la Didactique Appliquée : présence ou absence, horaire consacré, objectifs définis, contenu et compétences visées.

Un suivi pédagogique en séances de Didactique Appliquée a été mené sur 100 étudiants appartenant aux FUE-SVT et FUE-SVT- Métiers d'enseignement à l'ENS de Tétouan, promotions 2014, 2015 et 2016. Dans ce suivi, nous avons pu évaluer et quantifier (taux de réussite) la capacité des étudiants à maîtriser un ensemble de compétences définies comme nous avons relevé les principales difficultés rencontrées par les élèves professeurs lors de la préparation de certaines fiches ou supports scientifiques et pédagogiques 
ainsi que lors de la présentation des séquences pédagogiques ou leçons en situation de classe.

\section{Évaluation au niveau des établissements scolaires}

L'évaluation a été menée dans environ cent établissements scolaires de l'Enseignement Secondaire Collégial et Qualifiant à l'échelle nationale. Elle a été réalisée sur un échantillon de cent élèves professeurs en périodes de stage professionnel durant les années 2014, 2015 et 2016 et consiste à noter le même cours pris par l'élève en classe et à noter un ensemble d'informations pédagogiques sur une fiche d'observation en situation réelle de classe.

\section{RÉSULTATS}

\section{Évaluation des descriptifs des filières d'éducation}

D'après les données du Tableau 1, l'horaire consacré à la Didactique Appliquée est insuffisante; il a même connu une régression dans les filières accréditées après 2014 : passage de 90 à 50 heures, voire zéro heures dans certaines filières.

Dans la plupart des filières intégrant plus ou moins le module de didactique disciplinaire ou «didactique appliquée », le contenu n'est pas totalement conforme à la nouvelle vision de la Didactique Appliquée: compétences non parfaitement définies, confusion avec Didactique Générale avec parfois défragmentation de la Didactique Générale en différentes parties très loin de l'application avec considération dans certains cas de la Didactique Appliquée comme des séquences simples filmées au cours des séances du micro-enseignement.

Tableau 1. Horaire et contenu global de Didactique Appliquée dans différentes filières universitaires d'éducation accrédités ou en expertise

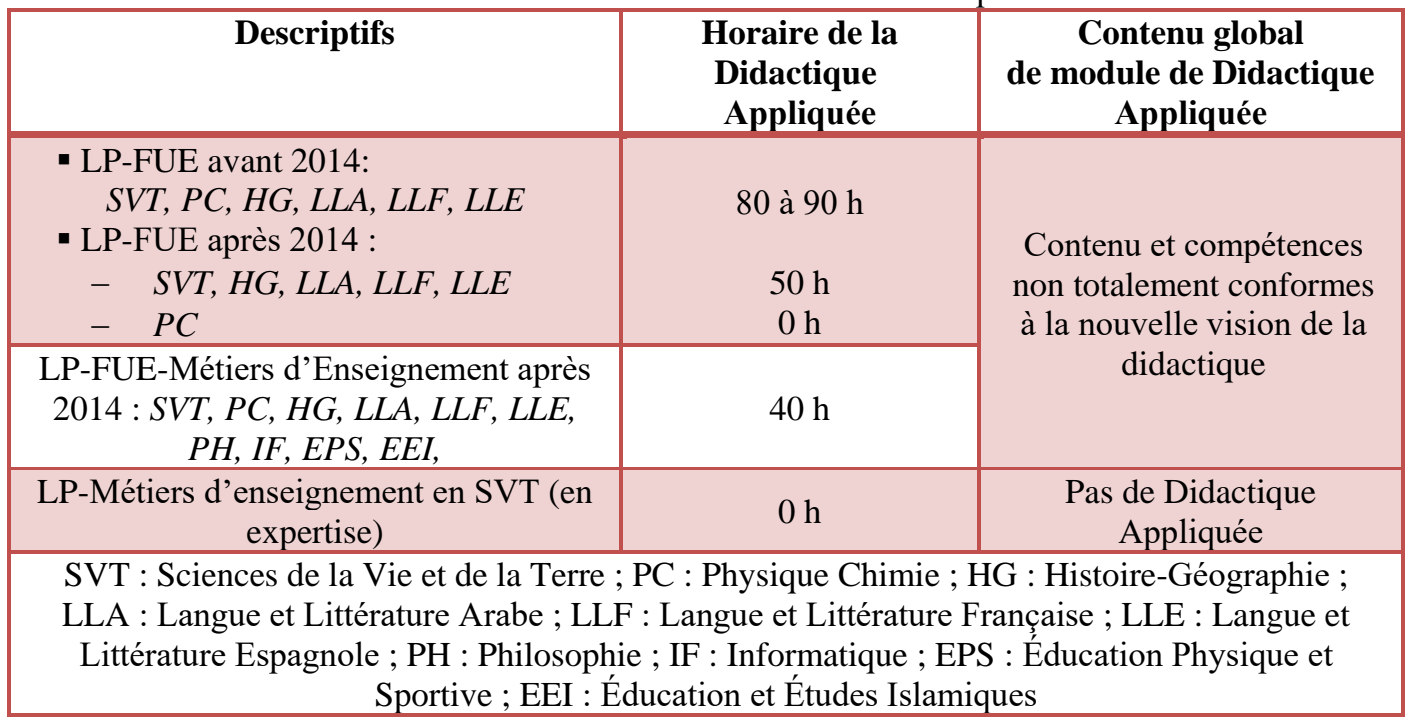



aux SVT

Évaluation des élèves professeurs en séances de Didactique Appliquée

Préparation de leçons : Au démarrage des séances de Didactique Appliquée, les élèves professeurs ignoraient totalement la méthodologie de préparation de leçons ; après plusieurs séances d'entraînement, plus que la moitié des élèves professeurs ont pu réussir la préparation des fiches scientifiques et fiches pédagogiques. Cependant, moins de $50 \%$ des élèves professeurs ont pu réussir la préparation des scénarios conceptuels, séquences pédagogiques et évaluation des élèves. Le Tableau 2 illustre parfaitement les différentes difficultés rencontrées par les étudiants.

Tableau 2. Difficultés rencontrées par les élèves professeurs lors de la préparation de leçons

SVT

\begin{tabular}{|c|c|c|}
\hline & $\begin{array}{l}\text { Taux de } \\
\text { réussite }\end{array}$ & Difficultés rencontrées \\
\hline $\begin{array}{c}\text { Fiche Scientifique } \\
\text { thématique en relation avec } \\
\text { la leçon }\end{array}$ & $52 \%$ & $\begin{array}{l}\text { - Recherche bibliographique } \\
\text { - Détermination des concepts de base } \\
\text { - Présentation et organisation des données }\end{array}$ \\
\hline Scénario conceptuel & $35 \%$ & $\begin{array}{l}\text { - Détermination précise des prérequis } \\
\text { - } \text { Identification des concepts scientifiques adaptés } \\
\text { au niveau à enseigner } \\
\text { - Reformulation des concepts en phrases adaptées } \\
\text { au niveau à enseigner } \\
\text { - } \begin{array}{l}\text { Planification pédagogique ou organisation } \\
\text { logique des concepts }\end{array}\end{array}$ \\
\hline Séquence Pédagogique & $30 \%$ & $\begin{array}{l}\text { - Choix des modalités d'investigation et du matériel } \\
\text { à utiliser } \\
\text { - Activités d'apprentissage : précision dans le choix } \\
\text { des verbes opérationnels } \\
\text { - Production des élèves et bilan }\end{array}$ \\
\hline $\begin{array}{l}\text { Fiche Pédagogique de la } \\
\text { leçon }\end{array}$ & $55 \%$ & $\begin{array}{l}\text { - Synthèse des données concernant la leçon } \\
\text { - Diversité des références scientifiques et } \\
\text { pédagogiques }\end{array}$ \\
\hline Évaluation des élèves & $40 \%$ & $\begin{array}{l}\text { - Modalités d'évaluation } \\
\text { - Innovation }\end{array}$ \\
\hline
\end{tabular}

Présentation orale de leçons : Le tableau 3 illustre parfaitement les difficultés rencontrées par les élèves professeurs lors de la présentation orale de leçons. Au terme des séances du module de Didactique Appliquée, la majorité des étudiants n'ont pas pu acquérir parfaitement les compétences et les objectifs visés dans cette activité (taux de réussite inférieur à $50 \%$ ). 
Tableau 3. Difficultés rencontrées lors de la présentation des leçons SVT

\begin{tabular}{|c|c|c|}
\hline & $\begin{array}{l}\text { Taux de } \\
\text { réussite }\end{array}$ & Difficultés rencontrées \\
\hline Tableau & $40 \%$ & $\begin{array}{l}\text { - Organisation du tableau } \\
\text { - Choix des illustrations conformes au tableau }\end{array}$ \\
\hline Plan de leçon & $45 \%$ & $\begin{array}{l}\text { - Répartition équilibrée des parties dans l'espace et dans le temps } \\
\text { - Pertinence de reformulation des titres et sous titres }\end{array}$ \\
\hline Introduction & $30 \%$ & $\begin{array}{l}\text { - Motivation et mobilisation des prérequis } \\
\text { - } \quad \text { Reformulation de la problématique }\end{array}$ \\
\hline $\begin{array}{l}\text { Matériel didactique et } \\
\text { informatique }\end{array}$ & $40 \%$ & $\begin{array}{l}\text { - Choix et exploitation du matériel } \\
\text { - } \quad \begin{array}{l}\text { Intégration pédagogique des outils informatiques } \\
\text { (vidéoprojecteur et TBI) }\end{array}\end{array}$ \\
\hline $\begin{array}{c}\text { Démarche pédagogique } \\
\text { et méthodes } \\
\text { d'enseignement }\end{array}$ & $30 \%$ & $\begin{array}{l}\text { - Application des approches pédagogiques } \\
\text { - Application des méthodes d'enseignement } \\
\text { - } \quad \text { Application de l'Enseignement Scientifique Fondé sur } \\
\text { l'Investigation (ESFI) } \\
\text { - Argumentation et transposition didactique } \\
\text { - Progression pédagogique }\end{array}$ \\
\hline Communication & $35 \%$ & $\begin{array}{l}\text { - Expression orale } \\
\text { - Communication graphique : schémas fonctionnels }\end{array}$ \\
\hline $\begin{array}{l}\text { Leçon supposée prise } \\
\text { par l'élève }\end{array}$ & $20 \%$ & $\begin{array}{l}\text { - Organisation de contenu } \\
\text { - } \quad \text { Synthèse des connaissances de base }\end{array}$ \\
\hline
\end{tabular}

\section{Évaluation au niveau des établissements scolaires}

L'évaluation en situation réelle de classe à travers 100 rapports de stage d'étudiants SVT, nous a permis de relever un ensemble d'anomalies dans les approches d'enseignement : préparation scientifique et didactique des leçons, productions pédagogiques, transposition didactique et construction du savoir scientifique. Le tableau 4 illustre parfaitement le degré d'anomalies qui entachent ces compétences de base.

Tableau 4. Évaluation didactique en situation réelle de classe SVT

\begin{tabular}{|c|c|}
\hline & Évaluation \\
\hline Fiche Scientifique de la thématique concernée & Jamais réalisée par plus de $98 \%$ des enseignants. \\
\hline Fiche Pédagogique de la leçon & Réalisée par plus de $50 \%$, mais souvent non conforme. \\
\hline Scénario conceptuel et séquences pédagogiques & Réalisés, mais non conformes par plus de $70 \%$. \\
\hline Modalités d'investigation & $\begin{array}{c}\text { Polycopié donné par plus de } 80 \% \text {. } \\
\text { Animations et vidéos utilisées par } 20 \% \text {. } \\
\text { Expérimentation et sorties sur le terrain presque } \\
\text { nulles. }\end{array}$ \\
\hline Supports pédagogiques préparés & Production originale faible $(25 \%)$ \\
\hline Production pédagogique des animations & Production très faible $(2 \%)$ \\
\hline Utilisation pédagogique des animations pédagogiques & Utilisation faible (20\%). \\
\hline Utilisation pédagogique du vidéoprojecteur & Anti-pédagogique dans plus de $60 \%$ des cas. \\
\hline Utilisation pédagogique du tableau & Anti pédagogique dans plus de $70 \%$ des cas. \\
\hline Utilisation du Tableau Blanc Interactif (TBI) & $\begin{array}{l}\text { Utilisation nulle : Absence des TBI dans la plupart } \\
\text { des cas. }\end{array}$ \\
\hline Leçon prise par les élèves & Anti-pédagogique dans plus de $80 \%$ des cas \\
\hline Références bibliographiques & $\begin{array}{l}\text { Limitées aux manuels scolaires marocains dans plus } \\
\text { de } 90 \% \text { des cas. }\end{array}$ \\
\hline
\end{tabular}




\section{DISCUSSION ET CONCLUSION}

La didactique est la base de la formation dans le métier d'enseignement d'une discipline. Elle vise beaucoup plus le développement des compétences pédagogiques des étudiants que l'acquisition des connaissances. Cette vision concorde avec la réforme de 2003 relative à l'Enseignement Supérieur marocain et avec certains travaux de recherche (Radi, 2017).

Pour cela, il est bien d'éclaircir la notion de didactique d'une part pour les étudiants et les enseignants exerçants le métier d'enseignement et d'autre part, pour les formateurs dans les centres de formation, les responsables et les conseillers pédagogiques dans les établissements scolaires.

La didactique des Sciences de la Vie et de la Terre ne se limite pas à une simple connaissance et mémorisation d'un ensemble de travaux de recherche en Didactique ou en Sciences d'Éducation; elle ne consiste pas uniquement à des séquences d'apprentissage filmées et superficiellement commentées; et ne se réduit non plus à un stage dans un établissement scolaire. La didactique, c'est l'art d'enseigner une discipline, c'est l'application pertinente en leçons et en leçons-TP de Biologie ou de Géologie ou toute autre matière enseignée. Chaque leçon construite demande une préparation méticuleuse, une présentation méthodique, une application pertinente de certains modèles théoriques et une évaluation rigoureuse. Chaque leçon ou TP bien présentée pourrait constituer un modèle ou un résultat d'application d'une recherche scientifique et didactique susceptible d'être un exemple à suivre.

Il est bien de préciser que la leçon passe par trois étapes fondamentales : Préparation, présentation et évaluation. La illustre et détaille plus ou moins ces étapes de construction et présentation d'une leçon.

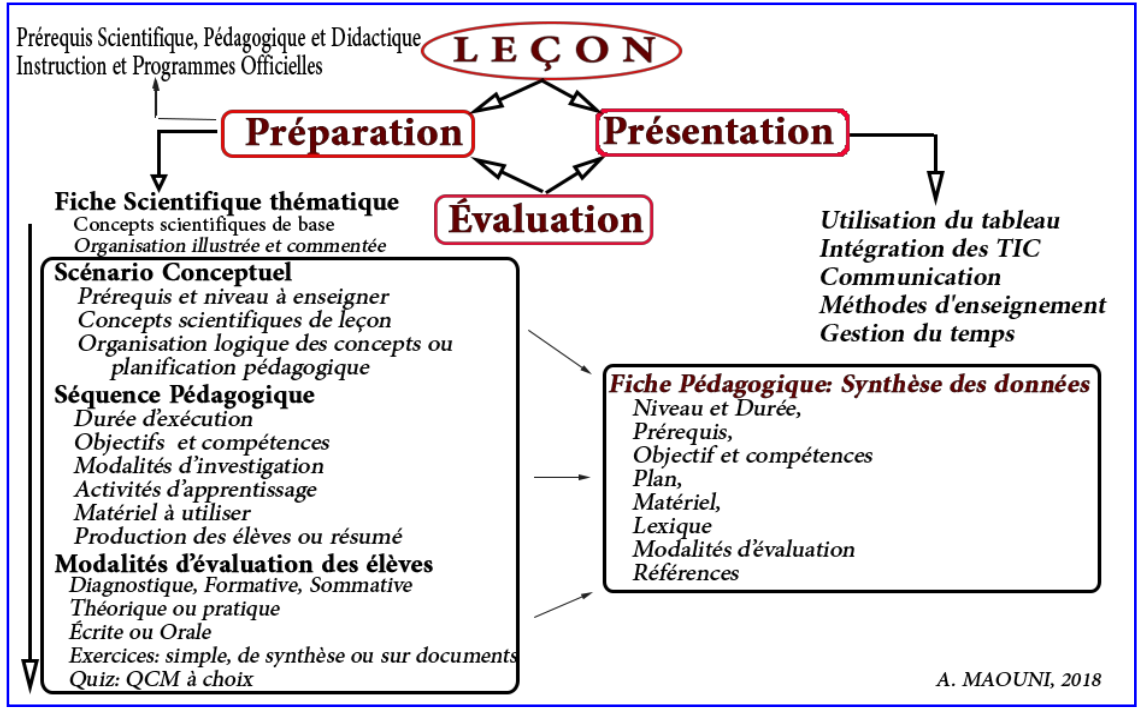

Figure 1. Principales étapes d'une leçon 
La nouvelle vision de la Didactique en SVT exige évidement de la part des élèves professeurs, une maîtrise du savoir scientifique, une formation théorique en Didactique et en Sciences d'Éducation, une connaissance des instructions et programmes officielles et principalement une formation pratique en Didactique. Elle exige également de la part des formateurs, une maîtrise du savoir et du savoir-faire scientifique, pédagogique, didactique et technologique (TICE). Dans les cursus de formation, la didactique pratique ou appliquée devrait suivre le cours théorique de la didactique et l'approfondissement des connaissances et précéder le stage professionnel. Son horaire devrait-être suffisant (120 heures au minimum pour une LP : 40h pour les préparations des fiches et $80 \mathrm{~h}$ pour les présentations et les discussions des leçons) et le nombre des étudiants en classe devrait être très réduit.

La préparation des fiches scientifiques est une étape primordiale dans la préparation des leçons : la plupart des formateurs ignorent cette phase et ne réalisent ni son sens ni son importance. La fiche scientifique n'est pas un cours, c'est une construction personnelle originale et synthétique en 3 à 5 pages maximum, traitant un savoir scientifique de la thématique en relation avec la leçon concernée, à partir de références non limitées à la langue maternelle d'enseignement. Il est impératif de signaler que les fiches scientifiques doivent répondre à des objectifs tels que définis dans l'ouvrage intitulé «Maîtriser l'information scientifique et médicale » (Febvre \& Giordan, 1990). Bien plus de la maîtrise de l'information scientifique et la compréhension de la documentation scientifique écrite, des compétences d'une grande importance dans le métier d'enseignement doivent également être acquises : développement de l'esprit de recherche bibliographique, développement de l'esprit de synthèse et de la capacité de distinction des connaissances de base scientifiques et enfin développement d'un savoir-faire constructif du savoir scientifique de base. Et c'est au niveau d'acquisition de ces compétences que la majorité des élèves professeurs rencontrent des difficultés. Ainsi le formateur en Didactique Appliquée doit être au niveau pour pouvoir évaluer et orienter correctement ces étudiants. Dans ce sens, et afin d'accomplir convenablement cette tâche d'encadrement de leçons ou leçons TP, certaines qualités sont nécessaires : expérience dans la classe, multidisciplinarité, haut niveau scientifique, compétences didactiques et compétences technologiques parfaitement acquises.

La construction du scénario conceptuel relatif à un sujet est principalement la clé de réussite de la préparation d'une leçon. Elle passe par quatre étapes : détermination précise des prérequis et de niveau à enseigner, identification des concepts scientifiques adaptés à un niveau à enseigner, reformulation des concepts en phrases adaptées au niveau à enseigner et enfin l'organisation logique des concepts ou planification pédagogique. Généralement les difficultés sont notées au niveau de l'identification des 
concepts et la planification pédagogique d'une leçon. Ceci pourra être expliqué par la présence de lacunes scientifiques et didactiques chez les élèves professeurs. Avec la préparation de fiches scientifiques et la multiplication de cette activité, certains élèves professeurs ont pu surmonter ces problèmes.

La séquence pédagogique est fondamentale dans la préparation d'une leçon. Elle consiste principalement à choisir et à préparer les modalités d'investigation, le matériel et les activités d'apprentissage afin de répondre à aux objectifs donnés dans le temps.

Il existe différentes modalités d'investigation ou supports pédagogiques d'apprentissage à exploiter en classe, mais le problème réside au choix d'une modalité par rapport à une autre. Il est préférable au départ de chercher un support pédagogique réel, sinon passer à un support numérique, et sinon encore passer à un support polycopié, sachant qu'il est possible de faire la combinaison de choix 3 avec le choix 1 ou 2. Les types et l'ordre de priorité de choix des supports pédagogiques sont illustrés dans la Figure 2 ci-dessous.

Figure 2. Diversité et choix des supports pédagogiques

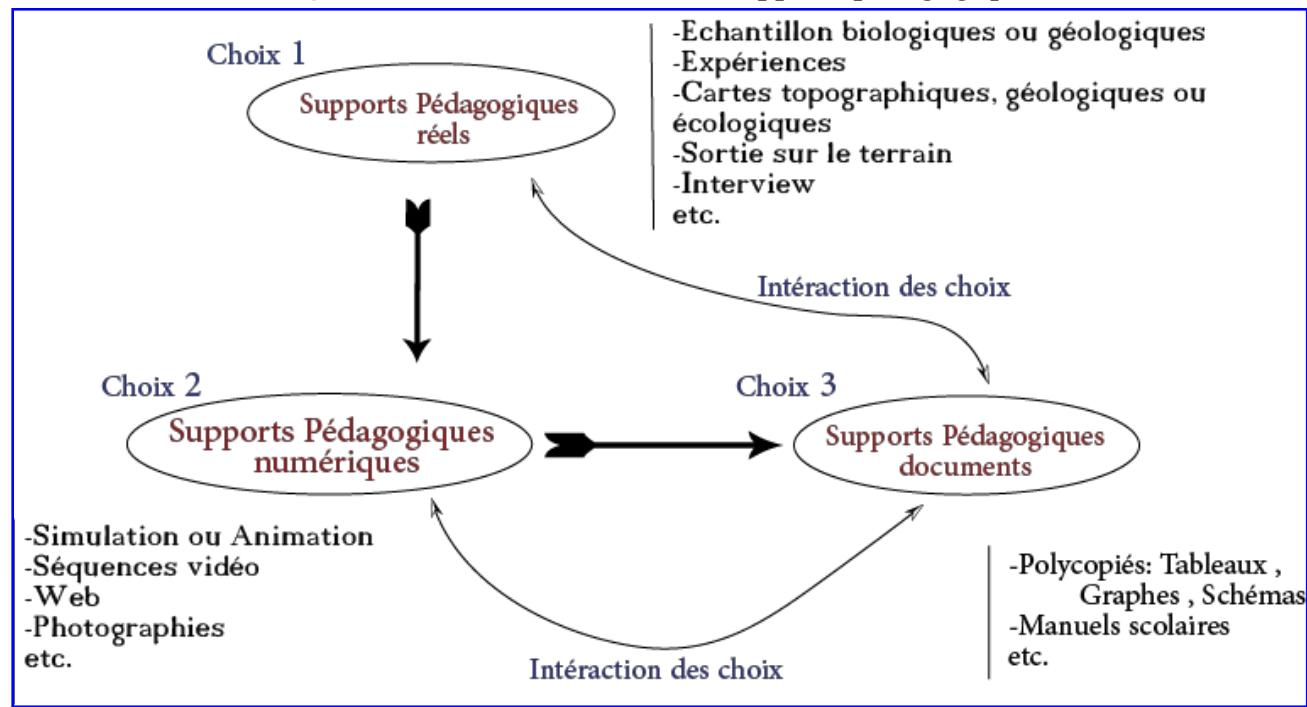

Un support pédagogique demande souvent une recherche pertinente, une transposition didactique suivant le niveau enseigné et la séquence pédagogique, et enfin demande de l'innovation didactique. Ce sont ces supports travaillés qui permettent aux élèves professeurs et généralement aux pratiquants du métier d'enseignement de rendre leurs apprenants plus actifs et plus participatifs dans la construction et la production du savoir.

Les activités d'apprentissage liées aux supports pédagogiques parfaitement préparés permettent aux élèves de développer leurs compétences. La taxonomie de Bloom trouve son application dans ces activités de l'élève par l'utilisation des verbes opérationnels : la plupart des enseignants exerçants 
ainsi que certains inspecteurs pédagogiques considèrent à tort, que ces activités incombent à l'enseignant, alors que c'est à l'élève d'y être en état d'activité, l'enseignant devant orienter tout simplement l'acte d'apprentissage. La production de l'élève est le résultat de ses activités personnelles ; elle se manifeste par des illustrations, des commentaires et des légendes de schémas, etc. Le bilan cognitif est une synthèse du phénomène étudié, il est établi par les élèves en collaboration avec le formateur : des résumés bien ciblés et des schémas fonctionnels sont appréciés dans un bilan cognitif.

La fiche pédagogique d'une leçon est la synthèse des données concernant une leçon, elle comporte un ensemble d'informations scientifiques et pédagogiques de la leçon qu'un élève professeur ou enseignant exerçant doit assimiler. C'est une fiche dynamique et non statique comme elle est considérée par la plupart des enseignants exerçants. Il est indispensable de rappeler les éléments constitutifs de cette fiche pédagogique : intitulé de la leçon, durée d'exécution, date de réalisation, établissement, cadre pédagogique, pré requis, objectifs cognitifs, compétences visées, plan de leçon, moyens matériels et didactiques, lexique des termes scientifiques clés et références bibliographiques. La motivation et la problématique de la leçon doivent-être prises en compte dans cette fiche. L'idéal est d'organiser et de présenter les éléments constitutifs de la fiche dans trois pages maximum. La plupart des étudiants rencontrent des problèmes essentiellement au niveau de la définition des objectifs cognitifs, de la reformulation des compétences visées et de l'établissement d'un plan de leçon équilibré dans l'espace et dans le temps. Ainsi, avec un entraînement continu sur plusieurs leçons et avec un bon encadrement, la plupart des élèves professeurs peuvent surmonter ces problèmes.

Le matériel utilisé en TIC est un outil éducatif puissant, son impact sur la qualité des apprentissages des étudiants et le développement professionnel des enseignants sont bien justifiés (Lebrun, 2011). Il est admis aujourd'hui qu'avec l'outil informatique, le «bon» enseignant en SVT ou l'enseignant «moderne» est celui qui est capable «d'apporter » la nature en classe. De même, il est capable de produire des supports didactiques adaptés au niveau de ses élèves (par exemple documents traités par Photoshop et séquences d'animations ou séquences vidéos traitées par des logiciels d'animation et de montage de film), capable encore d'utiliser des interfaces ExAO pour faire des mesures en SVT et capable également de se servir correctement d'un vidéoprojecteur et d'un Tableau Blanc Interactif (TBI) (Maouni et al., 2014). Il n'est plus acceptable aujourd'hui qu'en Didactique Appliquée un élève professeur arrive à terme de sa formation sans être parfaitement initié à l'exploitation convenable de l'outil informatique dans la construction et la présentation des leçons ou TP de Biologie ou de Géologie. Certes, les TIC sont actuellement mieux connues ; toutefois, la majorité des élèves professeurs et 
des enseignants exerçants ne maîtrisent pas les logiciels de base et exploitent ou intègrent de façon totalement anti-pédagogique l'outil informatique.

Le tableau noir ou blanc est un moyen didactique de valeur, souvent utilisé par les enseignants en classe ; cependant son exploitation n'est pas généralement pédagogique. À des fins utiles, il convient de rappeler le contenu et la fonction principale du tableau noir ou blanc en SVT (Tableau 5) : plan structuré et équilibré à gauche noté au fur et à mesure de la présentation de la leçon; lexique à droite soulignant les termes scientifiques clés (traduits si l'enseignement est en langue arabe) ; illustrations diversifiées et conformes au milieu du tableau. À la fin de la présentation, le tableau doit refléter toute la leçon, l'essentiel de la leçon et principalement les qualités pédagogiques et didactiques de l'enseignant qui a exposé la leçon.

Tableau 5. Organisation du tableau après présentation d'une leçon en SVT

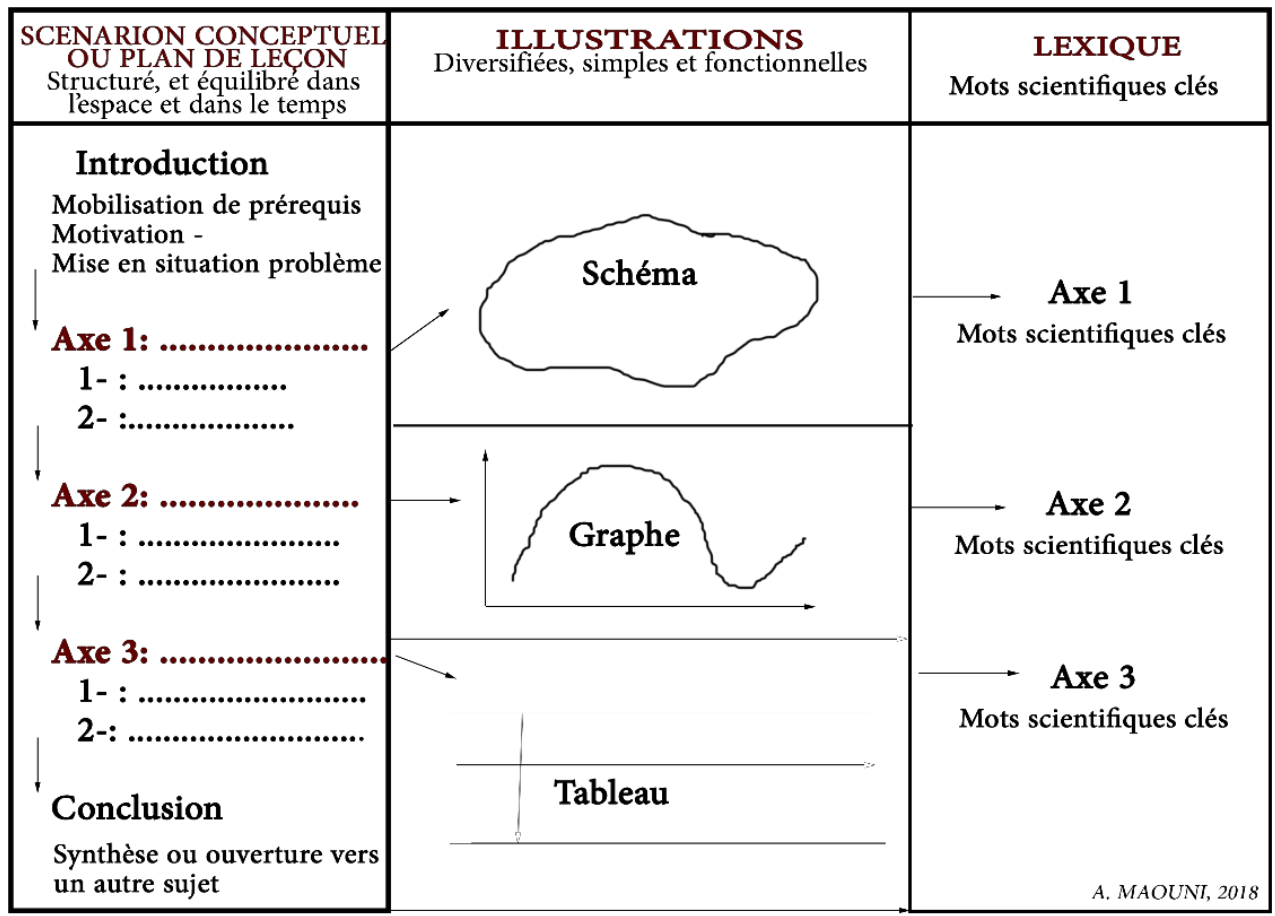

La désharmonie et la non complémentarité entre plan, illustrations et termes clés prouvent clairement l'utilisation anti-pédagogique du tableau par un enseignant en SVT. Il est regrettable qu'actuellement la plupart des enseignants exerçants dans l'Enseignement Secondaire marocain, utilisent le tableau de façon très confuse. Or, actuellement il est possible de combiner le vidéoprojecteur avec un tableau blanc : une projection directe sur un tableau blanc peut le rendre plus actif avec des possibilités d'écriture comme si on travaillait sur un TBI. 
Le TBI est actuellement un outil informatique plus efficace qu'un tableau classique grâce à l'interactivité qu'il mène. Toutefois, son absence dans la plupart des établissements secondaires marocains, nous laisse la possibilité d'exploiter les avantages du TBI : il suffit d'avoir un logiciel TBI, un vidéoprojecteur et une petite tablette pour pouvoir travailler comme si vous utilisiez un TBI.

La leçon SVT assumée par l'élève en classe de secondaire est devenue, dans les dernières années, un ensemble de données et de résultats d'expériences noyant les connaissances de base que l'élève est censé retenir à la fin de la leçon. Une leçon prise par l'élève ne montre pas uniquement une désorganisation des connaissances, mais reflète un ensemble de constats antipédagogiques dans notre système d'enseignement. Faute d'orientation didactique adéquate et de formations continues dans ce sens, des méthodes d'enseignement classiques et même des représentations pédagogiques et didactiques erronées continueront à peser dans notre système éducatif. Elle s'en suit gravement un transfert indirect de cet enseignement classique des enseignants exerçants vers les élèves professeurs stagiaires en absence d'un co-encadrement responsable et adéquat.

Enfin, la qualité de l'enseignement secondaire en SVT et autres disciplines ne peut s'améliorer s'il n y'a pas de rénovation des métiers de l'enseignement, de la formation et de la gestion (CESEFR, 2015). Cette rénovation comme nous la pensons à travers cet article pourrait avoir un impact positif sur l'amélioration de la qualité de notre enseignement secondaire à condition de l'appliquer en formation initiale et continue dans des centres de formation spécialisés dans la profession enseignante.

\section{References:}

1. CESEFR (2015). Conseil de l'Enseignement Supérieur de l'Education, de la Formation et de la Recherche. Vision Stratégique de la Réforme 2015-2030, pour une école de l'équité, de qualité et de la promotion. [Online] Available: http://www.csefrs.ma/default.aspx\#10.

2. Demounem, R. \& Astolfi,_J-P. (1996). Didactique des Sciences de la Vie et de la Terre : NATHAN Pédagogie.

3. Febvre, M. \& Giordan, A. (1990). Maîtriser l'information scientifique et médicale. DELACHAUX et NIESTLE 1990.

4. Lebrun, M. (2011). Impacts des TIC sur la qualité des apprentissages des étudiants et le développement professionnel des enseignants : vers une approche systémique. Rubrique de la revue STICEF, Volume 18, 2011, ISSN : 1764-7223, mis en ligne le 16/11/2011. [Online] Available: http://sticef.univ-lemans.fr/num/vol2011/03r lebruntice/sticef_2011_lebrun_03r.htm. 
5. Maouni, A., Mimet, A., Khaddor, M., Madrane, M. \& Moumene, M. (2014). L'intégration des TIC dans l'enseignement des SVT au Maroc : réalité et attentes. RADISMA, Numéro 10 (2014), 27 janvier 2014. [Online] Available: http://www.radisma.info/document.php ?id=1424. ISSN 1990-3219.

6. Martinand, J.L. (1994b). La Didactique des Sciences et de la Technologie et la Formation des Enseignants. ASTER, 19, pp. 61-75. [Online] Available : http://artheque.ens-cachan.fr/items/show/4666.

7. Ndong, L. (2011). Didactique des Sciences et Formation des Enseignants de Sciences de la Vie et de la Terre. RDST, 3 | 2011, 179208.

[Online] Available http://journals.openedition.org/rdst/420\#entries.

8. Radi, S. (2017). Pédagogie Active Et Développement Des Compétences Entrepreneuriales Des Étudiants : Analyse Empirique. European Scientific Journal September 2017. Edition Vol.13, No.26 ISSN: 1857 R 7881 (Print) e - ISSN 1857- 7431. 